\title{
Air Pollutant Emissions in the Fukui-Ishibashi and Nagel-Schreckenberg Traffic Cellular Automata
}

\author{
Alejandro Salcido, Susana Carreón-Sierra \\ Programa de Sustentabilidad Ambiental, Instituto Nacional de Electricidad y Energías Limpias, Cuernavaca, Mexico \\ Email: salcido@ineel.mx, susana.carreon@iie.org.mx
}

How to cite this paper: Salcido, A. and Carreón-Sierra, S. (2017) Air Pollutant Emissions in the Fukui-Ishibashi and $\mathrm{Na}$ gel-Schreckenberg Traffic Cellular Automata. Journal of Applied Mathematics and Physics, 5, 2140-2161.

https://doi.org/10.4236/jamp.2017.511175

Received: October 9, 2017

Accepted: November 7, 2017

Published: November 10, 2017

Copyright (c) 2017 by authors and Scientific Research Publishing Inc. This work is licensed under the Creative Commons Attribution International License (CC BY 4.0).

http://creativecommons.org/licenses/by/4.0/

\begin{abstract}
Vehicular traffic is a hard problem in big cities. Internal combustion vehicles are the main fossil fuel consumers and frame the main source of urban air pollutants, such as particulate matter, nitrogen oxides, and volatile organic compounds. Vehicular traffic is also a promoter of climate change due to its greenhouse gas emissions, such as $\mathrm{CO}$ and $\mathrm{CO}_{2}$. Awareness of the spatiotemporal distribution of urban traffic, including the velocity distribution, allows knowing the spatiotemporal distribution of the air pollutant vehicular emissions required to understand urban air pollution. Although no well-established traffic theory exists, some models and approaches, like cellular automata, have been proposed to study the main aspects of this phenomenon. In this paper, a simple approach for estimating the space-time distribution of the air pollutant emission rates in traffic cellular automata is proposed. It is discussed with the Fukui-Ishibashi (FI) and Nagel-Schreckenberg (NS) models for traffic flow of identical vehicles in a single lane. We obtained the steady-state emission rates of the FI and NS models, being larger those produced by the first one, with relative differences of up to $45 \%$ in hydrocarbons, $56 \%$ in carbon monoxide, and $77 \%$ in nitrogen oxides.
\end{abstract}

\section{Keywords}

Cellular Automata, Mobile Source Emissions, Traffic Emission Rates, Traffic Models, Fukui-Ishibashi, Nagel-Schreckenberg

\section{Introduction}

Big cities are suffering severe problems because of the growing number of vehicles moving over their streets. In Mexico City (CDMX), for example, the reg- 
istered vehicular fleet was estimated close to 5 million in 2015. Figure 1 describes information published by the Mexico's National Institute of Statistics and Geography (Instituto Nacional de Estadística y Geografía, INEGI) in relation to the increasing number of registered vehicles between 1980 and 2014 [1].

The vehicular fleet of CDMX is composed, in a great majority, by internal combustion vehicles that consume fossil fuels (gasoline, diesel, and gas); therefore, vehicular flow through the city streets is one of the main responsible for urban air pollution. In fact, the 2014 emissions inventory of CDMX [2] reported that the contributions of the mobile sources to the air pollutant emissions in the city were as described in Table 1 .

In Table 1, we observe that the vehicular traffic contributed with the $44 \%$ of the CDMX air pollution, in average. These emissions, of course, are not uniformly distributed in the region because there is no a uniform distribution of traffic in the city and winds either are not uniform either perennial. This means that the most polluted areas of a city are not necessarily those ones where more pollutants are released to the atmosphere.

Addressing the urban air pollution problems depends on the knowledge of the distribution modes of the urban vehicles in space, time, and over the possible speeds because these modes determine how the emissions of gases and particulate matter by the vehicles will result in a spatiotemporal distribution of emission rates in the city.

Once in the atmosphere, the air pollutants will be transported by the wind and dispersed by the atmospheric turbulence. Figure 2 shows the basic scheme of coupling of the models required for simulating the impacts of traffic emissions on air quality. It comprises a traffic flow model, which estimates the spatiotemporal distribution of the vehicles and their velocity distribution; an emission model, which allows determining the spatiotemporal distribution of the emission rates of the pollutants produced by the traffic flow; an atmospheric

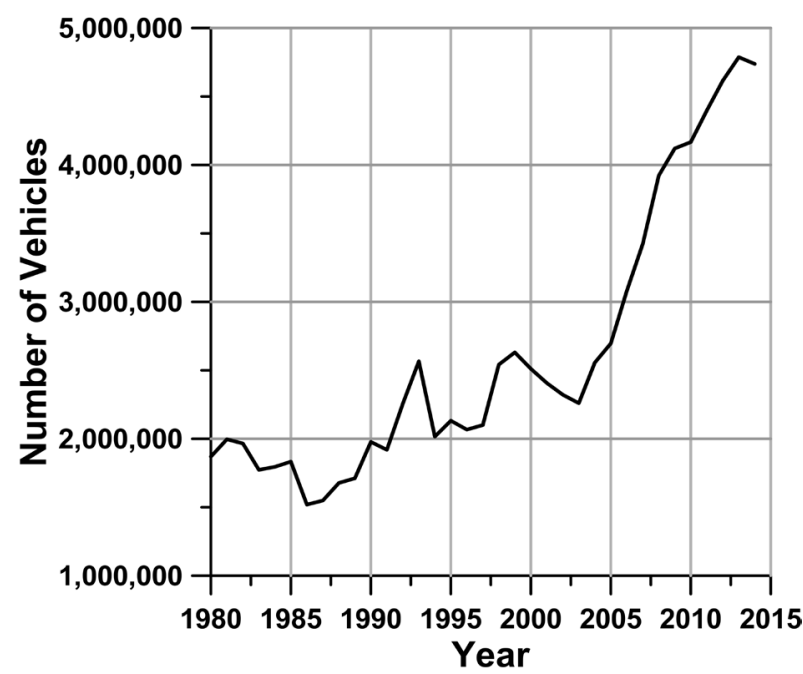

Figure 1. Growing of the MCMA's vehicular fleet from 1980 to 2014 . 


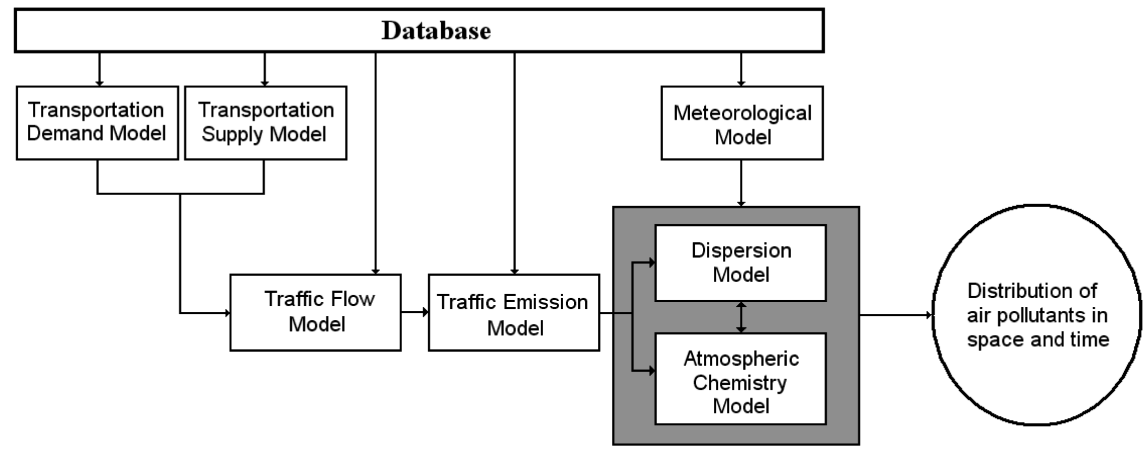

Figure 2. Basic scheme of coupling of the models required for simulating the impacts of the emissions of mobile sources on air quality.

Table 1. Mobile source air pollutant emissions [\%] according to the 2014 emissions inventory of CDMX.

\begin{tabular}{cccccccccccc}
\hline $\mathrm{PM}_{10}$ & $\mathrm{PM}_{2.5}$ & $\mathrm{SO}_{2}$ & $\mathrm{CO}$ & $\mathrm{NO}_{\mathrm{x}}$ & $\mathrm{TOC}$ & $\mathrm{VOC}$ & $\mathrm{CO}_{2}$ & $\mathrm{~N}_{2} \mathrm{O}$ & $\mathrm{HFC}$ & $\mathrm{CO}_{2 \text {-eq }}$ & Black Carbon \\
\hline 20.7 & 28.7 & 16.5 & 96.1 & 78.5 & 11.3 & 20.0 & 61.5 & 50.0 & 98.0 & 49.0 & 83.7 \\
\hline
\end{tabular}

transport and dispersion modelling system of the emissions; and an atmospheric chemistry model, which addresses the possible transformations of the pollutants in the atmosphere.

There is no a complete theory for traffic flow phenomena. However, several models and approaches for analyzing traffic phenomena, such as traffic jamming and some other common modes of traffic flow, have been developed from the macroscopic, mesoscopic and microscopic standpoints.

The scientific treatment of the traffic flow phenomena began with Robert Herman in 1956, and some years later, in the early 1960s, Herman and Prigogine started to study vehicular traffic as a collective flow phenomenon, developing a kinetic theory for multi-lane traffic flow using a Boltzmann like model for the vehicle interactions [3].

In the second half of the 1980s, an alternative line of research emerged for traffic flow simulation based on cellular automata [4], but its proper development started in the early 1990s with the models proposed by K. Nagel and M. Schreckenberg [5] and by M. Fukui and Y. Ishibashi [6], hereafter referred as NS and FI models, respectively. They defined cellular automata models for the microscopic simulation of vehicular traffic. The initial NS and FI models were formulated for identical vehicles moving on a single lane highway. In these models, each vehicle can be at rest or be hopping from site to site in a 1D lattice with a positive integer speed which does not exceed a given maximum. The dynamic rules of these cellular automata are probabilistic and control the propagation, acceleration, and braking of the model vehicles, although conserving its number and preventing them from collisions and overtaking. Several variants and extensions of the NS and FI basic models have been developed for simulating traffic flow in double-sense and multi-lane highways [7] [8] [9], and also for 
2D complex traffic networks similar to that of a city [10] [11] [12] [13] [14].

In this paper, we propose a simple approach for estimating the spatiotemporal distribution of the emission rates for the traffic flow phenomena described by the NS and FI models. This approach assumes it is possible to know the velocity distribution of the system, i.e. how many vehicles are moving with each one of the possible velocities (from zero to a maximum speed). The velocity distribution of the traffic cellular automata can be obtained always by computer simulations, but also theoretically, at least for a class of models. In this paper, for obtaining the velocity distributions, we used computer simulations and the statistical mechanics approach proposed in [15] [16] [17] for the maximum entropy states of 1D traffic cellular automata. The results of this work show that due to the transition rules of the FI model, which favor the highest speeds, the steady state emission rates of this model are higher than those ones of the NS model, with relative differences as large as $45 \%$ for hydrocarbons, $56 \%$ for carbon monoxide, and $77 \%$ for nitrogen oxides.

The rest of the paper is organized as follows. In Section 2, we described our methodological approach. First, we presented the basic NS and FI traffic cellular automata, discussing, in particular, how the velocity distributions of these models can be obtained from computer simulations and from the theoretical approach proposed by Salcido and collaborators [15] [16] [17]. We described also the estimation model for the pollutants emission rates from mobile sources [18], and the extension we proposed for traffic cellular automata. In Section 3, we presented and discussed the results of the application of our methodological approach to the problem of estimating the distribution of the pollutant emissions from the NS and FI traffic cellular automata.

\section{Methodology}

In this section, we provide first a brief introduction to cellular automata; then we describe and discuss the NS and FI traffic cellular automata models and the approaches to frame their velocity distributions, and, finally, we present the approach to estimate the model cars emissions.

\subsection{Cellular Automata}

Cellular automata (henceforth: CA) are a class of spatially and temporally discrete, complex dynamical systems characterized by local interaction and an inherently parallel form of evolution. Following a suggestion of Stanislaw Ulam, cellular automata were first introduced by John von Neumann in the early 1950s to act as simple models of biological self-reproduction [19]. Cellular automata can be considered as prototypical models for complex systems and processes consisting of a large number of identical, simple, locally interacting components [20]. The study of CA has generated great interest over the years because of their ability to generate a rich spectrum of very complex patterns of behavior out of sets of relatively simple underlying rules [20] [21] [22] [23]. Moreover, CA ap- 
pear to capture many essential features of complex self-organizing cooperative behavior observed in real systems.

There exists a wide variety of particular CA models; however, most of them usually possess the following common generic characteristics. The system substrate consists of a one-, two- or three-dimensional lattice of cells; all cells are equivalent; each cell takes on one of a finite number of possible discrete states; each cell interacts only with cells that are in its local neighborhood; and at each discrete time step, each cell updates its current state according to a transition rule taking into account the states of cells in its neighborhood.

If $\psi(x, t)$ denotes the state at cell $x$ at time $t, V(x)$ is the neighborhood of this cell (in a well-defined sense of proximity), and $\{\psi(\tilde{x}, t) \mid \tilde{x} \in V(x)\}$ is the set of the states of the cells in the neighborhood, then the state at cell $x$ at time $t+1$ will be given by

$$
\psi(x, t+1)=F(\{\psi(\tilde{x}, t) \mid \tilde{x} \in V(x)\})
$$

Here $F$ represents the transition rules of the system dynamics. Note that both the neighborhood and the transition rule have the same definitions for all the lattice cells. Usually, neighborhoods contain the first nearest neighbors (von Neumann), or the first and second nearest neighbors (Moore). Some widely known cellular automata are the Wolfram's 1D elementary cellular automata [24] and the Conway's Game of Life [25].

\subsection{One-Dimensional Traffic Cellular Automata}

The basic one-dimensional traffic cellular automata (B1DTCA) are concerned with the traffic flow of identical vehicles (cars) on a single lane highway with no anticipation. This class of CA models shares the following properties:

- The system can be considered as a lattice gas of $\mathrm{N}$ indistinguishable unit mass particles, which evolves in a 1D lattice with $L$ cells (or sites).

- The particles of the system obey an exclusion principle, which establishes that no more than one particle can be in one lattice cell.

- Each particle can be at rest or be moving with a positive integer velocity that cannot exceed a given maximum $v_{\max }>0: v_{k}=k$ with $k=0,1,2,3, \cdots, v_{\max }$. This means that the particles move always in the same direction (say, from left to right), and never can go in the reverse direction. The velocity $v_{\max }$ is interpreted as a speed limit that drivers have to respect inexcusably.

- The dynamics of the system is defined by a set of local transition rules. The same rules are applied simultaneously to all the lattice cells. These rules allow no particle collisions neither overtaking. Traffic accidents never occur and each car follows always same another car.

- The local transition rules preserve the number of particles, but not necessarily momentum neither the energy.

- The system evolution occurs in discrete time steps. Time increases in one unit only once all the cells of the system have been updated according to the 
transition dynamical rules.

In Figure 3, we illustrate a possible spatial distribution of the system cars in the lattice. Here, the different car velocities are evidenced with different background colors. It must be noted that the no anticipation condition implies that each car with velocity v occupies $v+1$ lattice sites.

The distance among adjacent cells is usually defined as the unit, but for the purpose of real traffic simulations, it is assumed to be the average front-bumperto-front-bumper distance of adjacent vehicles under conditions of strongly jammed traffic and set equal to $7.5 \mathrm{~m}$. In this case, the time step is set equal to one second, and the velocity increases in steps of $27 \mathrm{~km} / \mathrm{h}$.

We can describe the state of the system indicating the number of lattice cells $(L)$, the total number of particles $(N)$, and the numbers of particles $N_{k}$ which move with velocity $v_{k}=k \quad\left(k=0,1,2, \cdots, v_{\max }\right)$. In general, however, we will use the intensive properties (densities) defined as

$$
n=\frac{N}{L}, \quad n_{k}=\frac{N_{k}}{L}
$$

The density of particles (i.e. the number of particles per cell) is equal to the sum of the partial densities

$$
n=\sum_{k} n_{k}
$$

and the densities of momentum (traffic flow) and kinetic energy are given by

$$
q=n v=\sum_{k} v_{k} n_{k}, \quad \varepsilon=\sum_{k} \varepsilon_{k} n_{k}
$$

respectively, where $v$ is the average speed of the traffic flow and $\varepsilon_{k}=v_{k}^{2} / 2$ is the kinetc energy of a particle with speed $v_{k}$.

The traffic models developed by Nagel and Schreckenberg [5] and by Fukui and Ishibashi [6] [26] belong to the class of B1DTCA.

\subsubsection{The Nagel and Schreckenberg Model}

The dynamics of the Nagel-Schreckenberg model [5] is defined by the following set of local transition rules. If one vehicle is located at the cell $c(c=1,2,3, \cdots, L)$ at time $t$, and it is moving with velocity $v(c, t)$, then

- Rule 1. Acceleration: $v(c, t)$ is replaced by $u(c, t)=\min \left\{v(c, t)+1, v_{\max }\right\}$.

- Rule 2. Braking: $u(c, t)$ is replaced by $w(c, t)=\min \{d(c, t), u(c, t)\}$, where $d(c, t)$ is the number of empty cells ahead the cell $c$, at time $t$.

- Rule 3. Randomization: the velocity of the vehicle located at cell $c$ is updated to $v(c, t+1)=\max \{w(c, t)-1,0\}$ with probability $p$, or to $v(c, t+1)=w(c, t)$ with probability $1-p$.

- Rule 4. Flow: the vehicle jumps from cell $c$ to cell $c+v(c, t+1)$.

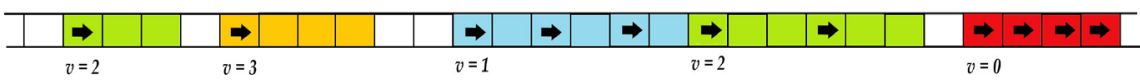

Figure 3. One instantaneous spatial distribution of the system cars. Colors make evident the possible different car velocities. 
These rules are applied simultaneously to all the non-empty lattice cells; time increases by one only when all the lattice cells have been updated.

These rules have widely accepted simple interpretations. Rule 1 mimics the fact that drivers like to go as fast as allowed. Rule 2 takes into account that one driver has to reduce its car's velocity to avoid the collision against the vehicle ahead. Rule 3 aims to take into account some effects which produce velocity fluctuations, even in the free flow case; for example, the road conditions (slopes, potholes, and speed humps, among others), the impact of climatic conditions on traffic flow, and psychological effects. Consequently, this rule can produce braking overreaction, which may give rise to spontaneous jamming [27]. Finally, Rule 4 displaces the vehicles in the lattice. It is worthy of comment that Brilon and $\mathrm{Wu}$ [28] have questioned Rule 3; they argue that it has no theoretical basis. Nevertheless, Rule 3 is essential in simulating realistic traffic flow since otherwise the model dynamics would be completely deterministic [5].

Collectively, these four rules enable the NS model to reproduce the basic phenomena of real traffic, such as the occurrence of the phantom traffic jams. These rules define a minimal model in the sense that any further simplification of them no longer produces nontrivial and realistic behavior. For proper modelling of the fine structure of traffic, however, it is necessary the introduction of additional rules and/or the modification of the transition rules above-presented.

\subsubsection{The Fukui and Ishibashi Model}

In the Fukui-Ishibashi model [6] [26] [29] the cars can move by at most $v_{\max }$ lattice sites in one time step if vehicles in front do not block them. Specifically, if at time $t$ the number of empty sites $h$ in front of a car is larger than $v_{\max }$ then, in the next time-step, it can move forward $v_{\max }$ sites with probability $1-p$, or $v_{\max }-1$ sites with probability $p$. Here, the randomization probability $p$ represents the degree of stochastic delay. Within the framework of this model, drivers do not like to use brakes if they are far away from the vehicle ahead. For a large density of cars, the stochastic delay in the FI model represents the assurance of the avoidance of crashes. When the stochastic delay is null ( $p=0)$, this cellular automaton is referred to as the deterministic FI model with the maximum velocity $v_{\max }$. On the other hand, the case $p=1$ defines the deterministic FI model with the maximum velocity $v_{\max }-1$. If $h<v_{\max }$ at time $t$, then the car can only move by $h$ sites in the next time-step. Important differences of the FI model with respect the NS model are that the acceleration of cars may occur abruptly and that stochastic delay only affects the high-speed cars.

\subsubsection{The Maximum Entropy States}

Many of the cellular automata models proposed for traffic flow are based on the NS and FI models that we described in the previous sections. These models, in general, have been developed as computational systems for simulating traffic phenomena, and there are no analytical theoretical formulations to describe them. In fact, up today, very few efforts have been made to establish a unified 
theoretical formalism for the traffic cellular automata. In this section, we provide a brief description of a statistical mechanics' analysis carried out by Salcido et al. [15] [16] [17] for obtaining the equilibrium states of the B1DTCA (such as the NS and FI models) from a maximum entropy principle.

It is important to stress that the dynamical rules of the models like NS and FI are not microscopically reversible (they do not satisfy the principle of detailed balance [30] [31]), and, consequently, the system is always far from equilibrium. In fact, the Nagel-Schreckenberg and Fukui-Ishibashi models have been considered as variants of the well-known asymmetric exclusion process (ASEP), a paradigm of non-equilibrium systems [32]. In spite of this fact, an entropy function can be defined for the class of B1DTCA, and the velocity distribution that corresponds to the maximum-entropy states may be determined [15] [16] [17].

Such as detailed in [17], we assume that our system belongs to the class of B1DTCA (defined in Section 2.2). In addition, we assume that it has periodic boundary conditions, so that when one particle leaves the lattice by one end, it appears immediately in the other end. Moreover, it is observed that each particle of the system, which is moving with the velocity $v_{i}$ can be considered as a block that occupy $v_{i}+1$ cells in the $1 \mathrm{D}$ lattice. This observation allows showing that the entropy per cell of the system of blocks is

$$
s=(\lambda+n) \ln (\lambda+n)-\lambda \ln (\lambda)-\sum_{i} n_{i} \ln \left(n_{i}\right)
$$

where $\lambda$ is the vacancy (the number of cells per cell that remain empty after accommodating all the blocks of the system in the lattice) and $n_{i}$ is the partial density of the particles with velocity $v_{i}$ (the number of blocks each one occupying $v_{i}+1$ cells, per cell of the system). We observe that

$$
\lambda=1-\sum_{i}\left(v_{i}+1\right) n_{i} \geq 0
$$

Equations (3) and (4) give the densities of particles, momentum, and kinetic energy of the system.

Under this context, the maximum entropy states of the system are given by

$$
n_{i}=\lambda\left(\frac{\lambda}{\lambda+n}\right)^{v_{i}} \mathrm{e}^{-\alpha-\beta \varepsilon_{i}}=n_{0}\left(\frac{\lambda}{\lambda+n}\right)^{v_{i}} \mathrm{e}^{-\beta \varepsilon_{i}}
$$

Here $\alpha$ and $\beta$ are Lagrange multipliers, and it has been defined $n_{0} \equiv \lambda \mathrm{e}^{-\alpha}$ [17]. Equation (8), for each velocity $v_{i}=v_{1}, v_{2}, \cdots, v_{\max }$, gives the number of particles (cars) per cell which are moving with that velocity; i.e., this equation gives the velocity distribution of the system. This maximum entropy approach describes, as a particular case, the low-density behavior of the FI model with a very good agreement [17], and also reproduce, approximately, at least, the steady states of the NS model [15] [16] [17].

\subsection{Pollutant Emission Rates of Traffic Cellular Automata}

Let us assume that $e\left(\alpha, v_{i}\right)$ is the emission rate of the pollutant $\alpha$ of one particle (a model car of a traffic cellular automaton) which is moving with veloc- 
ity $v_{i}$. Let us assume also that at the cell $x$, the average number of particles per cell which are moving with the velocity $v_{i}$ at time $t$, is $n_{i}(x, t)$. Then, the partial emission rate of the pollutant $\alpha$ due to the vehicles with velocity $v_{i}$ is given by

$$
\mu\left(\alpha, v_{i}, x, t\right)=e\left(\alpha, v_{i}\right) n_{i}(x, t)
$$

And the total emission rate of the pollutant $\alpha$ at time $t$, due to all the particles of the system is

$$
Q(\alpha, t)=\sum_{x} \sum_{i} \mu\left(\alpha, v_{i}, x, t\right)=\sum_{x} \sum_{i} e\left(\alpha, v_{i}\right) n_{i}(x, t)
$$

where the sums extend over all the lattice cells and over all the possible velocities.

For traffic cellular automata, the velocity distributions that we need to estimate their pollutant emissions can be obtained in general from computer simulations, but also from a theoretical standpoint such as the maximum entropy approach that we described in the previous section.

The emission rate $e\left(\alpha, v_{i}\right)$, on the other hand, must be determined experimentally, using emission factors, or with a proper emission model. This function represents a subset of a mobile source emission inventory disaggregated by pollutant, type of vehicle, and speed of movement of the vehicle. This emission rate, of course, will depend also on the characteristics and conditions of the vehicle, on driving habits, and on the weather conditions. In general, the reference data for estimating the emissions of road vehicles is obtained by measuring the emissions of a representative vehicle in a controlled ambient and simulating specific driving condition. The results of the observations are usually aggregated either by estimating a functional relationship (e.g., the German recommendations for economic assessment of road infrastructure investments (EWS) [33]) or by clustering the data into typical driving situations (e.g., the Workbook on Emission Factors for Germany and Switzerland [18]).

The EWS has the advantage that the full functional relationship on the vehicle's velocity $v$ is given for a specific pollutant $\alpha$ and vehicle type [34]:

$$
e_{f}(\alpha, v)=\left\{\begin{array}{l}
c_{0}+c_{1} v^{2}+\frac{c_{2}}{v} \text { for } v>20 \mathrm{~km} / \mathrm{h} \\
\min \left\{c_{S G},\left(c_{0}+c_{1} v^{2}+\frac{c_{2}}{v}\right)\right\} \text { for } v \leq 20 \mathrm{~km} / \mathrm{h}
\end{array}\right.
$$

with parameters $c_{0}, c_{1}$ and $c_{2}$ for free flow, and parameter $c_{S G}$ for stop-andgo traffic conditions. These parameters are differentiated by vehicle type and pollutant. A reduction factor is applied for each pollutant in order to take account of advanced pollution reduction technologies. From the emission factor, $e_{f}(\alpha, v)$, the emission rate $e(\alpha, v)$ is calculated as follows:

$$
e(\alpha, v)=\frac{e_{f}(\alpha, v) v}{3600}
$$

Here, the emission rate is expressed in $[\mathrm{g} / \mathrm{s}]$ if the velocity and the emission 
factor are expressed in $[\mathrm{km} / \mathrm{h}]$ and $[\mathrm{g} / \mathrm{km}]$, respectively.

Extending EWS [33] [34], we assume that the emission factor and the emission rate of the pollutant $\alpha$ for a particle with velocity $v_{i}$ in traffic cellular automata, can be estimated as

$$
\begin{gathered}
e_{f}\left(\alpha, v_{i}\right)=A_{0}(\alpha)+A_{1}(\alpha) v_{i}^{2}+\frac{A_{2}(\alpha)}{v_{i}}+\frac{A_{3}(\alpha)}{\sqrt[5]{v_{i}}} \\
e\left(\alpha, v_{i}\right)=B_{0}(\alpha)+B_{1}(\alpha) v_{i}+B_{2}(\alpha) v_{i}^{3}+B_{3}(\alpha) \sqrt[5]{v_{i}^{4}}
\end{gathered}
$$

for $v_{i}=v_{0}, v_{1}, \cdots, v_{\max }$. The parameters $A_{r}$ and $B_{r}$ depend on the pollutant $\alpha$ and on the characteristics of the vehicle. The parameter $B_{0}$ represents the emission rate of one vehicle at rest (stopped, but with its motor running). Note the additional term in Equation (13) in comparison with Equation (11). In Section 2.5 , we will see that this term allows a very good fitting to the available data reported in [34].

\section{Results and Discussion}

The main goal of this work is to estimate and compare the emissions rates of the Nagel-Schreckenberg and Fukui-Ishibashi traffic cellular automata. For simplicity, we considered only simulations of the steady states for models with $v_{\max }=5$ and randomization probability $p=0.25$. They were carried out with an 800 -cells lattice with periodic boundary conditions. Particle densities from 0 to 1 in steps of 0.01 were considered. In each simulation, the system was allowed to evolve during 600 time steps, starting from an initially random spatial distribution of the particles. The simulation was repeated 1000 times for each particle density value. In this case, the ensemble average of the local velocity distribution at each lattice cell is the same as the ensemble average of the global one.

\subsection{The Velocity Distributions}

In Figure 4 and Figure 5, we present some results of the space-time evolution of the NS and FI models, which we obtained from computer simulations. In Figure 4, it is shown the evolution of the NS model for particle densities $n=0.10,0.12,0.17$ and 0.30 . Each row (horizontal line) contains an instantaneous spatial distribution of the particles. Time increases vertically from top to bottom. It is observed that $n=0.12$ defines a transition between two different flow regimes: from free to congested flow.

Figure 5 shows the spatiotemporal evolution of the FI model for densities $n=0.18,0.20,0.22$ and 0.30 . In this case, $n=1 / 5$ defines a transition between the free flow and congested flow regimes.

The graphs of Figure 6 show, for the NS and FI models ( $v_{\max }=5, p=0.25$ ), the steady state partial densities $n_{0}, n_{1}, \cdots, n_{5}$ of the particles with velocities $v_{0}, v_{1}, \cdots, v_{5}$, respectively; and the densities of kinetic energy, $\varepsilon$, and momentum, $q$, and the velocity $v$, of the traffic flow, expressed as functions of the vehicular density $n$. The partial densities $n_{i}(n)$ were obtained as ensemble averages 


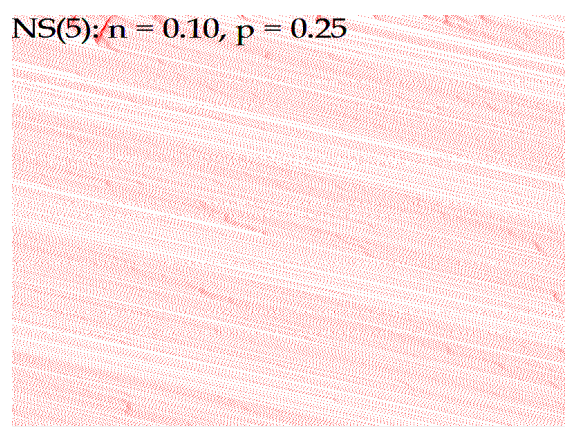

(a)

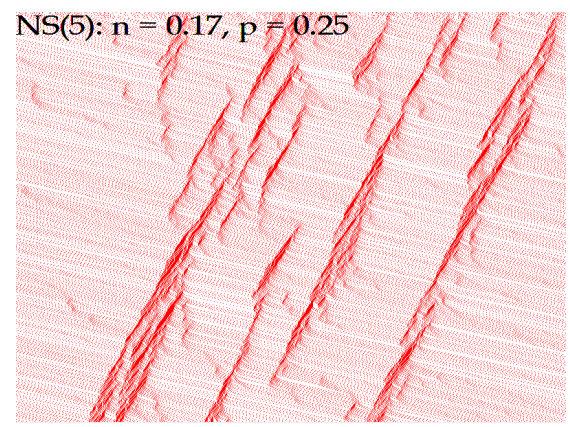

(c)

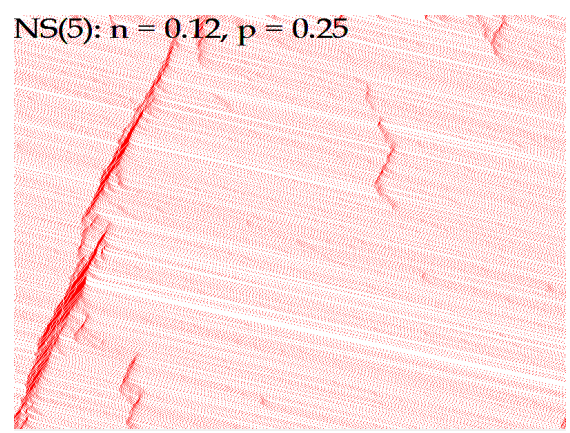

(b)

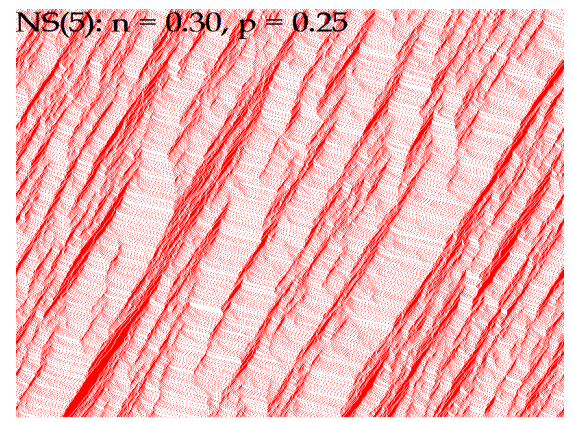

(d)

Figure 4. Computer simulations with the NS model with $v_{\max }=5$ and $p=0.25$, for several values of the particle density. In particular, for $n=0.12$, a transition between two different flow regimes is observed. (a) $n=0.10$; (b) $n=0.12$; (c) $n=0.17$; (d) $n=0.30$.

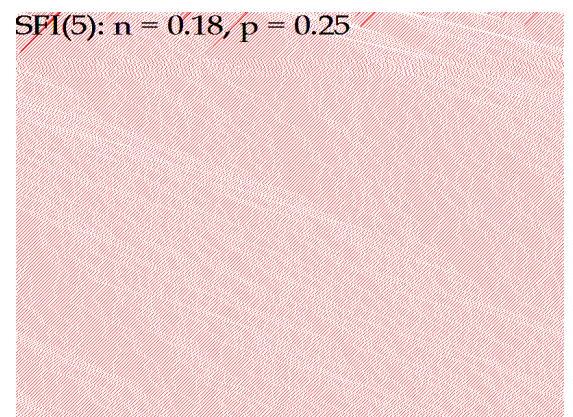

(a)

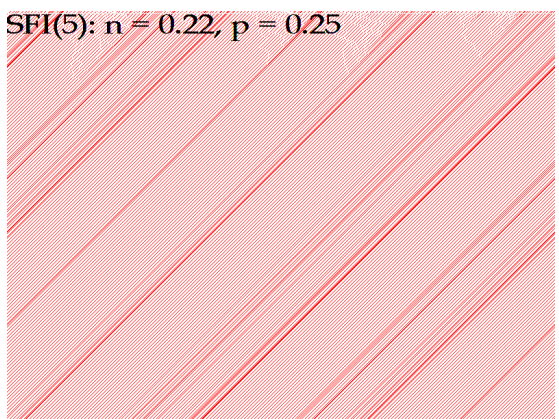

(c)

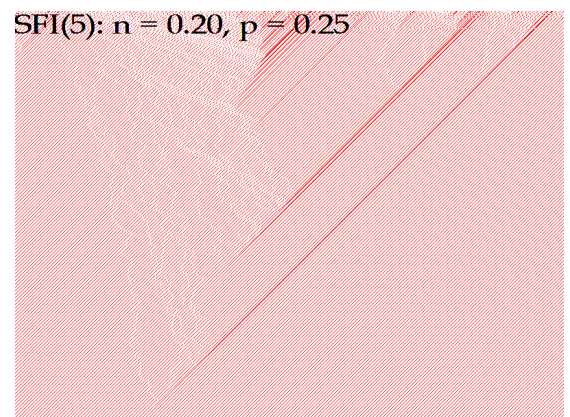

(b)

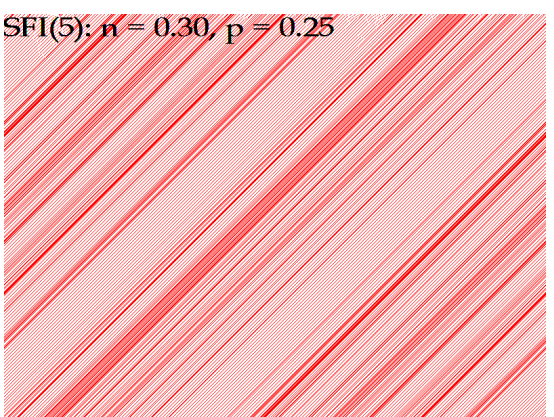

(d)

Figure 5. Computer simulations of the FI model with $v_{\max }=5$ and $p=0.25$, for several values of the particle density. A transition from the free to the congested flow is observed around $n=1 / 5$. (a) $n=0.18$; (b) $n=0.20$; (c) $n=0.22$; (d) $n=0.30$. 


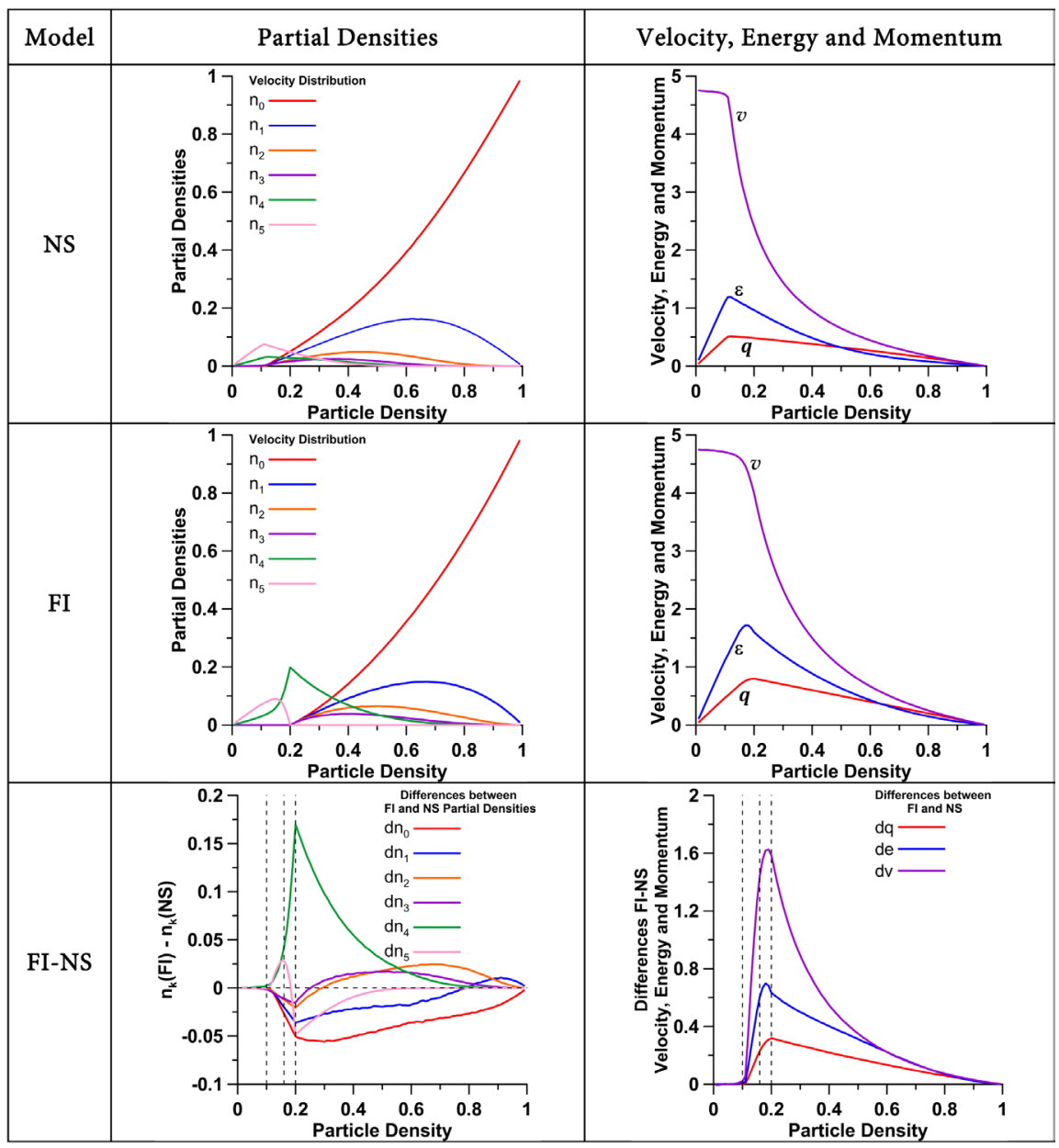

Figure 6. Steady state properties of the NS and FI models with $v_{\max }=5$ and $p=0.25$. The partial densities $n_{0}, n_{1}, \cdots, n_{5}$ and the densities of kinetic energy $\varepsilon$, and momentum $q$, and the velocity $v$ of the traffic flow, are shown in the first and second rows. The last row shows the differences of these properties between the FI and NS models.

over the 1000 repetitions of the simulations. The properties $\varepsilon, q$, and $v$ were calculated from Equations (4). In the traffic science jargon, the plot of $q$ is known as the fundamental diagram. In the bottom row, we presented graphs which show the differences of these properties between the FI and NS models.

Here, it is observed that all the partial densities, $n_{0}, n_{1}, \cdots, n_{5}$ of the NS model are different from zero in the interval $0<n<1$, although only the partial densities $n_{4}$ and $n_{5}$ have non-negligible values in the interval $0<n<0.12$. For the FI model, otherwise, only the partial densities $n_{4}$ and $n_{5}$ are greater than zero in the low-density regime $0<n<1 / 5$, and for $1 / 5<n<1$, all partial densities, except $n_{5}$, are different from zero. Then, for $v_{\max }=5$ and $p=0.25$, the free flow regime in the FI model extends up to densities close to $n=1 / 5$, while in the NS model this regime extends only up to $n=0.12$. This is clear in the plots of the average velocity of the traffic flow and in the densities of momentum and kinetic energy, which are shown in the right column of Figure 6. 
The left column of the last row of Figure 6 shows the plots of the differences between the partial densities of the FI and NS models:

$$
d n_{k}(n)=n_{k}(N I, n)-n_{k}(N S, n), \quad k=0,1, \cdots, 5
$$

These plots show that these differences are negligible in the interval $0<n<0.12$, that the partial densities $n_{4}$ and $n_{5}$ in the FI model are larger than in the NS model in the density interval $0.12<n<0.18$, but in the same interval the partial densities of the smaller velocities are larger in the NS model than in the FI model. For particle densities $0.18<n<0.26$, only the partial density $n_{4}$ of the FI model is larger than in the NS model, and for $0.3<n<1$, the numbers of particles with velocities $v_{2}, v_{3}$, and $v_{4}$ are larger in the FI model. In the high density region $0.79<n<1$, also the number of particles with velocity $v_{1}$ is larger in the FI model than in the NS model. These observations underline that, in general, the average velocity of traffic flow is larger in the FI model, such as it is shown in the plots we presented in the right column of the last row of Figure 6. These results are consequences of the dynamical rules of the FI traffic cellular automaton, where the particles can increase their velocities faster than in the NS model, and where the stochastic delay only applies to the high-speed cars. As we will show in Section 3.2, this behavior has an important consequence in relation with the air pollutant emissions of the traffic flows described by these models.

For concluding this section, in the graphs of the Figure 7 we showed the maximum entropy states of the NS and FI models for the same set of couples of particle density and kinetic energy per cell, $(n, \varepsilon)$, of the simulations we described previously for these traffic models. Again, the partial densities $n_{0}, n_{1}, \cdots, n_{5}$, and the densities of kinetic energy $\varepsilon$ and momentum $q$, and the velocity $v$, of the traffic flow, are shown. The partial densities presented in this figure (left column) were obtained by numerical solution of Equation (8) using the points $(n, \varepsilon)$ of the curves $\varepsilon(n)$ presented in the first and second rows of the right column of Figure 6, as input data. An exception was the case of the low density behavior of the FI model, $0<n<1 / 5$, because the following exact analytical solution of Equation (8) exists for this case [17]:

$$
\begin{gathered}
n_{5}=\frac{1}{2}\left\{1-4 n-\sqrt{(1-4 n)^{2}-4 n(1-5 n)(1-p)}\right\} \\
n_{4}=n-n_{5} \\
v=4+\frac{1}{2 n}\left\{1-4 n-\sqrt{(1-4 n)^{2}-4 n(1-5 n)(1-p)}\right\}
\end{gathered}
$$

For each model, important differences can be observed between the plots of the partial densities obtained from computer simulations (first and second rows of Figure 6) and from the maximum entropy approach (Figure 7), mainly for the high-density regimes. The main reason for these behavior differences is due to the dynamical transition rules of the NS and FI traffic cellular automata, which do not satisfy the principle of detailed balance [30] [31], and, therefore, both systems are always driven out of equilibrium. 


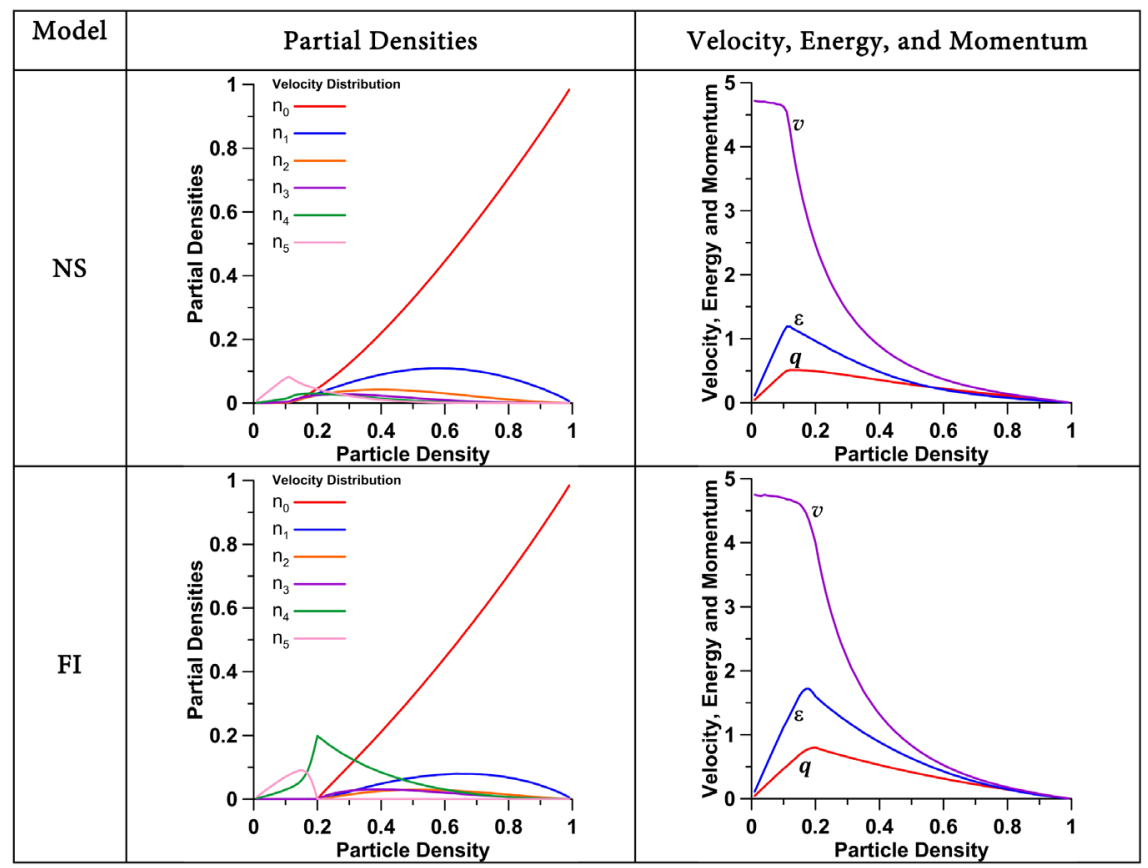

Figure 7. Maximum entropy states of the NS and FI models with $v_{\max }=5$ and $p=0.25$. The partial densities $n_{0}, n_{1}, \cdots, n_{5}$, the velocity $v$, and the densities of kinetic energy $\varepsilon$, and momentum $q$, of the traffic flow, are shown.

\subsection{The Emission Rates}

With reference to Section 2.2, we underline that the distance among adjacent cells is usually assumed as the average front-bumper-to-front-bumper distance of adjacent vehicles under conditions of strongly jammed traffic, and it is set equal to $7.5 \mathrm{~m}$. Then, if the time step is set equal to one second, the velocity of a vehicle will change in steps of $27 \mathrm{kph}$. Therefore, when comparing with real traffic data, the interpretations of the model velocities will be as follows,

$$
v_{0}=0, v_{1}=27, v_{2}=54, v_{3}=81, v_{4}=108 \text { and } v_{5}=135 \mathrm{kph}
$$

The emission factors we used in this work are based on [18]. In the data base, the emission factors are given for traffic situations which are characterized by a mean speed (beside other dependencies). In order to obtain an effortless mapping between the velocity and the amount of emission, the different traffic situations were aggregated into bins of size $10 \mathrm{~km} / \mathrm{h}$ [34] [35].

Figure 8 shows the emission factors and the emission rates for three different pollutants: carbon monoxide (CO), hydrocarbons ( $\mathrm{HC})$, and nitrogen oxides $\left(\mathrm{NO}_{\mathrm{x}}\right)$.

In this figure, we observed that the emission behavior is rather different for the distinct pollutants, and that their amount strongly depends on velocity. For estimating the pollutant emissions in the NS and FI traffic cellular automata, we used the emission rates of $\mathrm{CO}, \mathrm{HC}$, and $\mathrm{NO}_{\mathrm{x}}$ shown in Figure 8. The associated best fitting functions and their determination coefficients $\left(R^{2}\right)$ are presented in Table 2. 


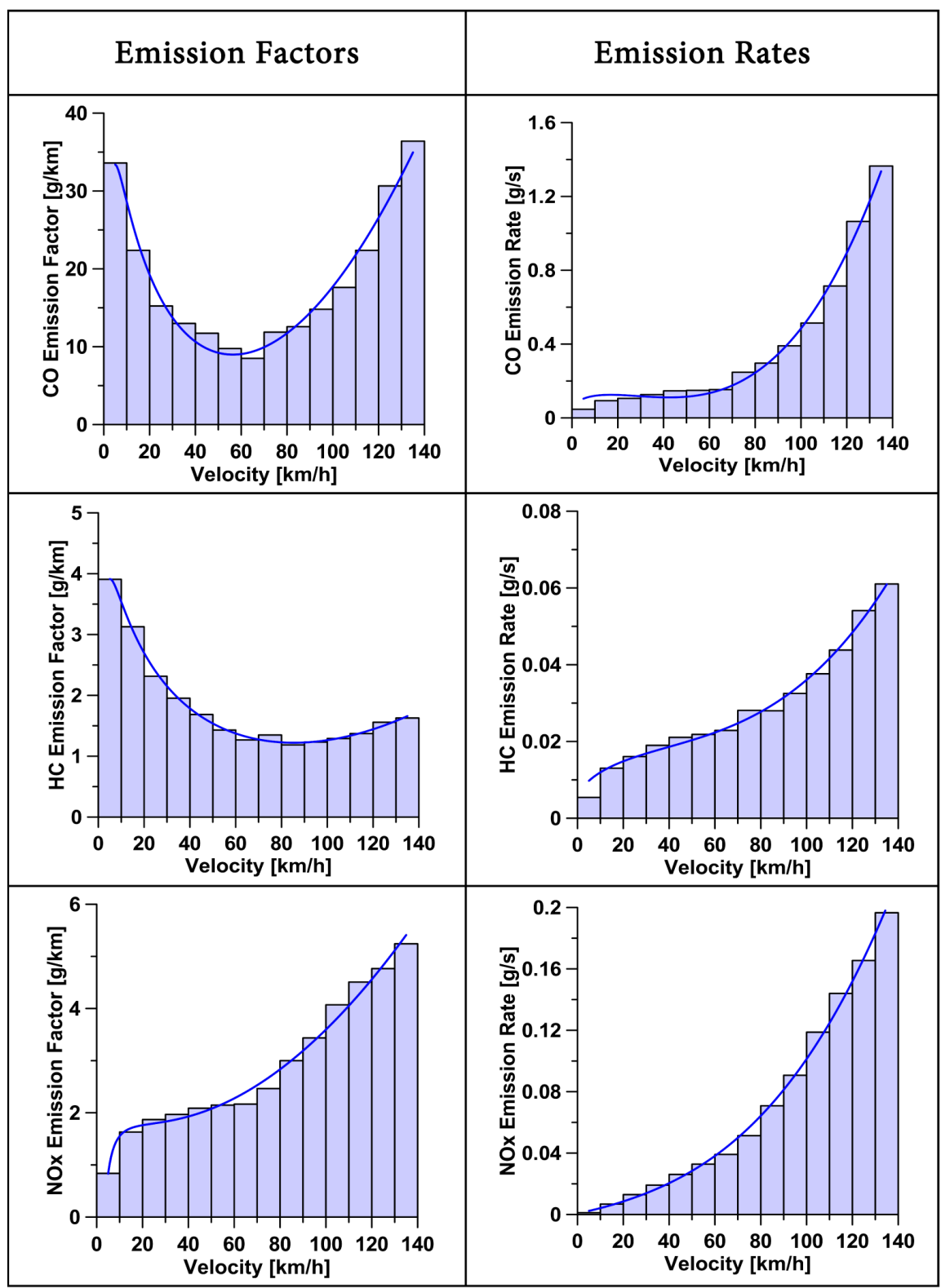

Figure 8. Vehicular emission factors and emission rates for the pollutants CO, HC, and $\mathrm{NO}_{\mathrm{x}}$ as functions of velocity. We observe that the amount of emission is strongly dependent on the vehicle's velocity and on the kind of pollutant. The best fitting curves (blue solid lines) of the model functions (13) and (14) to the data available in [34] [35] are also shown.

Figure 9 shows the partial emission rates produced by the simulations with the NS and FI models for vehicle emissions of carbon monoxide (CO), hydrocarbons (HC), and nitrogen oxides $\left(\mathrm{NO}_{\mathrm{x}}\right)$. The differences between these models are also shown. These results were obtained with the Equation (9), using the NS and FI velocity distributions shown in Figure 6, and the $\mathrm{CO}, \mathrm{HC}$ and $\mathrm{NO}_{\mathrm{x}}$ emission rates given in Table 2 with the allowed particle velocities given by the Equation (17).

The differences between the emission rates produced by the FI and NS models 
(Figure 9) are shown in Figure 10. Here we note that the FI traffic model produces the larger emission rates, particularly those associated with the velocity $v_{\max }-1$. However, when the system is in the high-density regime, the NS model produces an emission rate larger than the FI model does associated with the largest velocity $v_{\max }$.

In Figure 11, we show the partial emission rates obtained with the maximum entropy velocity distributions (see Figure 7). In Figure 12, the differences between the FI and NS emission rates are shown.

The graphs of Figure 9 and Figure 11 show important qualitative similarities between the partial emission rates estimated with the velocity distributions obtained by computer simulation and by means of the maximum entropy approach. However, there exist non-negligible numerical differences which are reflected also in the total emission rates, particularly for the $\mathrm{CO}$ and $\mathrm{HC}$ pollutants, as it is shown in the Figure 13.

In Figure 13, we present the total emission rates for the models NS and FI. It includes the results obtained with computer simulations and with the maximum

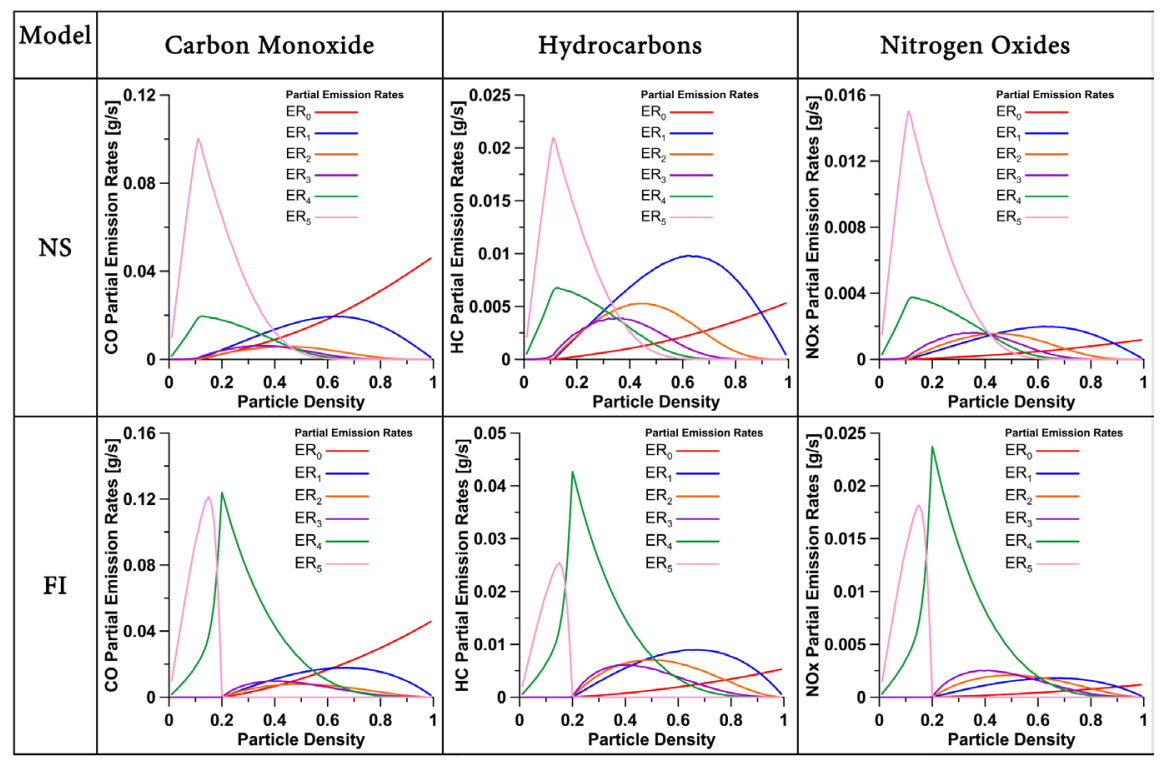

Figure 9. Partial emission rates of the NS and FI traffic models for the pollutants CO, HC and $\mathrm{NO}_{\mathrm{x}}$.

Table 2. Best fitting functions for the traffic emission rates of $\mathrm{CO}, \mathrm{HC}$, and $\mathrm{NO}_{\mathrm{x}}$ as dependent on the vehicle velocity. Estimated from data available in [34] [35]. Determination coefficients $\left(R^{2}\right)$ are also shown.

\begin{tabular}{ccc}
\hline Pollutant & Emission Rate $[\mathrm{g} / \mathrm{s}]$ & $\boldsymbol{R}^{2}$ \\
\hline $\mathrm{CO}$ & $e_{\mathrm{CO}}\left(v_{i}\right)=0.0467-0.020966 v_{i}+7.551701 \times 10^{-7} v_{i}^{3}+0.044694 \sqrt[5]{v_{i}^{4}}$ & 0.991621 \\
$\mathrm{HC}$ & $e_{\mathrm{HC}}\left(v_{i}\right)=0.0054-0.000810 v_{i}+1.931618 \times 10^{-8} v_{i}^{3}+0.002321 \sqrt[5]{v_{i}^{4}}$ & 0.988483 \\
$\mathrm{NO}_{\mathrm{x}}$ & $e_{\mathrm{NO}_{\mathrm{x}}}\left(v_{i}\right)=0.0012+0.000703 v_{i}+5.577680 \times 10^{-8} v_{i}^{3}-0.000653 \sqrt[5]{v_{i}^{4}}$ & 0.996947 \\
\hline
\end{tabular}




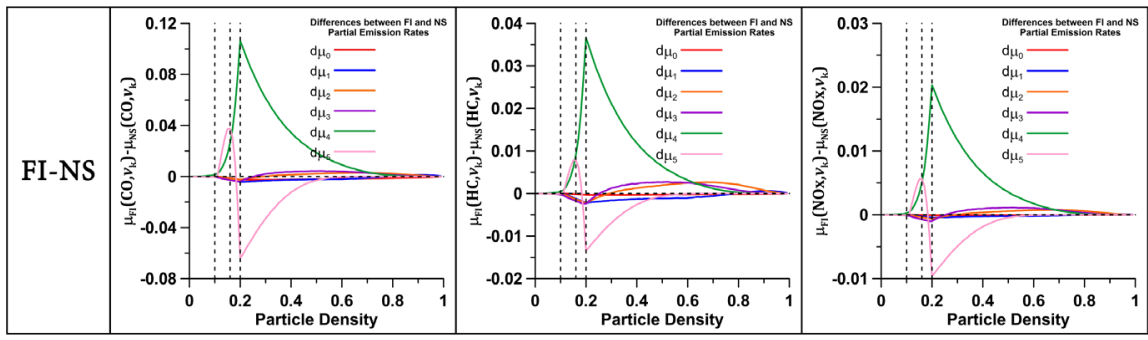

Figure 10. Differences between the FI and NS partial emission rates of Figure 9.

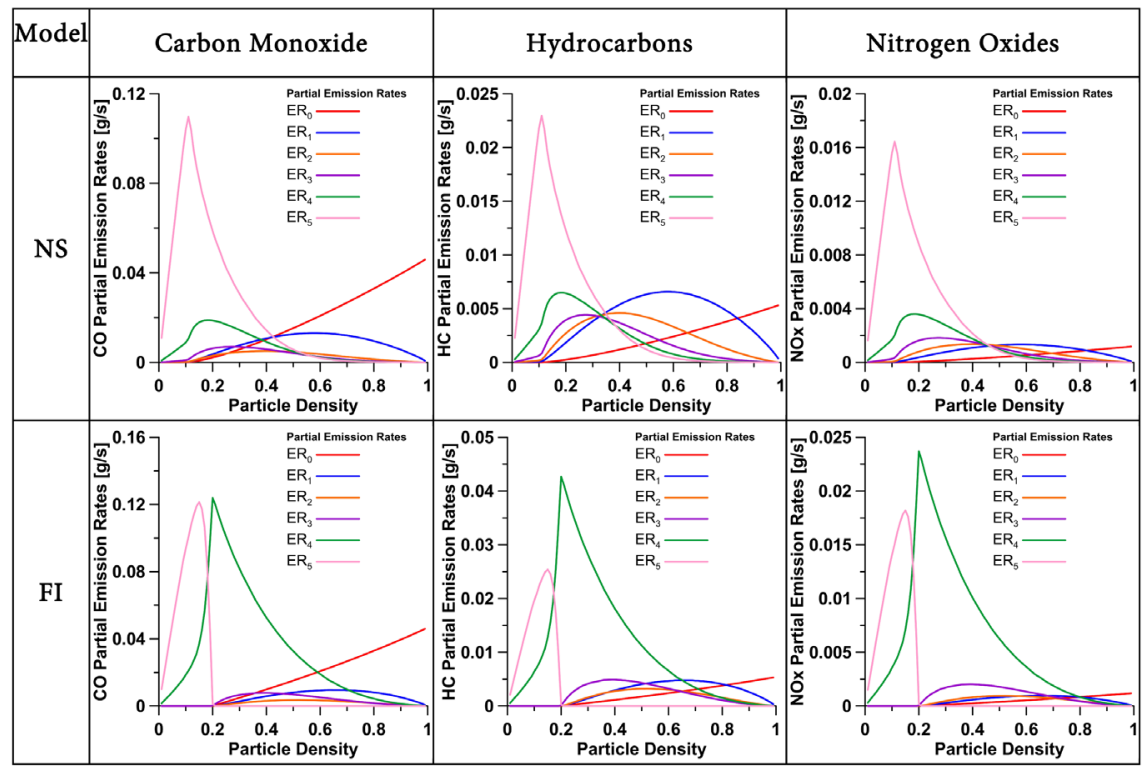

Figure 11. Partial emission rates of the NS and FI traffic cellular automata estimated with the maximum entropy velocity distribution.

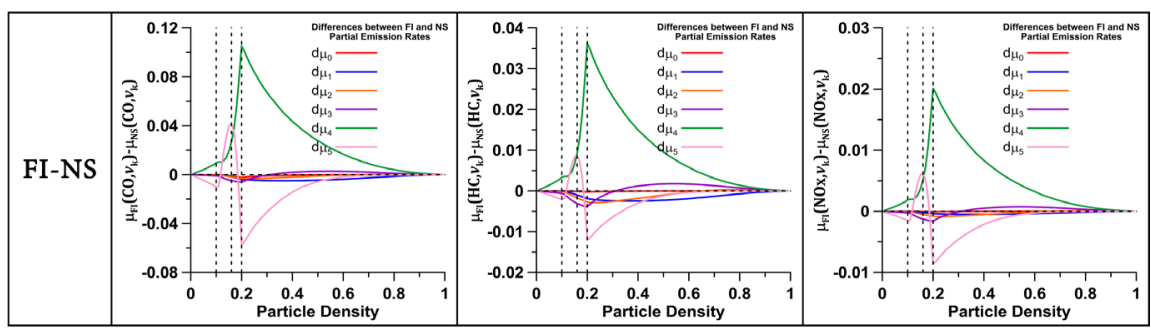

Figure 12. Differences between the FI and NS emission rates of Figure 11.

entropy approach. The plots presented in Figure 13 were obtained by summing, respectively, the partial emission rates of Figure 9 and Figure 11, such as it is indicated by the Equation (10).

In Figure 13, we observe: for densities $0<n<0.11$, both traffic cellular automata produced the same total emission rates for each pollutant; for densities $n>0.11$, the FI traffic model produced total emission rates of $\mathrm{CO}, \mathrm{HC}$ and $\mathrm{NO}_{\mathrm{x}}$ larger than the NS model did, respectively. In the limit $n \rightarrow 1$, the emission rates of both models become the same because all the particles become at rest, remaining only the emissions in the idle conditions. 


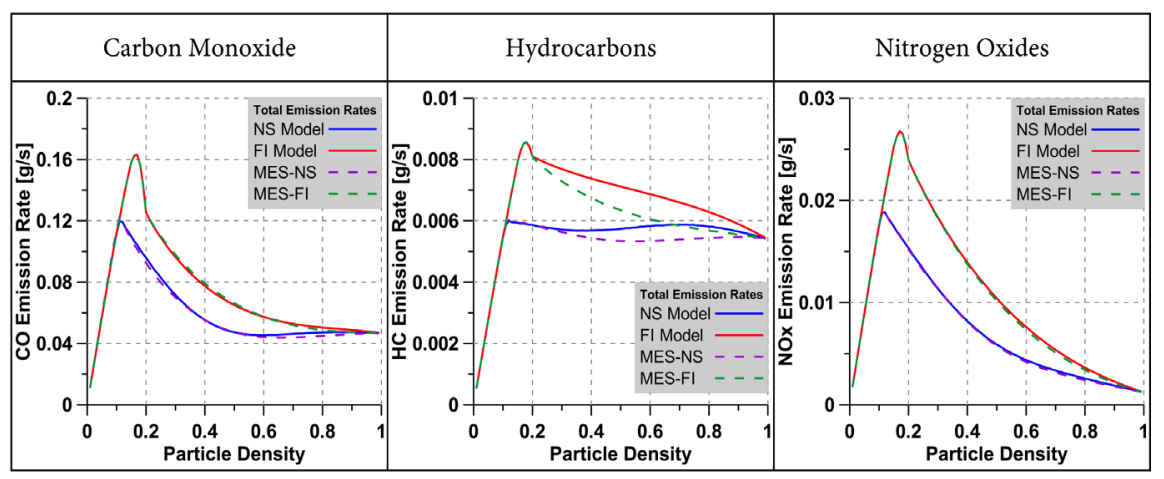

Figure 13. Total Emission rates of the NS and FI traffic cellular automata ( $v_{\max }=5$ and $p=0.25$ ) as functions of the particle density. The solid line curves correspond to the computer simulations of steady state conditions. The dotted line curves correspond to the maximum entropy states.

Figure 13 shows also the plots of the emission rates estimated with the maximum entropy state velocity distributions. We observe that the deviations with respect the estimations with velocity distributions obtained from computer simulations only result important in the case of the hydrocarbons, because in this case the differences and the emission rates themselves are of the same order. The larger emission rates of the FI model are a consequence of its dynamic rules because the stochastic delay is applied only to the highest speed vehicles, extending its free flow regime up to particle densities higher than in the NS model.

In Figure 14, the relative differences

$$
\delta(\alpha, n) \equiv 100\left(\frac{Q_{F I}(\alpha, n)-Q_{N S}(\alpha, n)}{Q_{N S}(\alpha, n)}\right),
$$

between the total emission rates of the FI and NS models for steady state conditions and the selected pollutants (Figure 13), are shown. Here we can underline three interesting density intervals:

1) $0<n<0.11$ : This is the interval of the low density behavior of the FI and NS traffic models with $v_{\max }=5$ and $p=0.25$. Here, almost all the particles are moving with one of the two highest velocities, $v=5$ (i.e. $v_{\max }$ ) or $v=4$ (i.e. $\left.v_{\max }-1\right)$. It is a free flow regime. In this density region, the relative differences between the estimations of the emission rates of the NS and FI models are negligible for all the pollutants we considered:

$$
\delta(\mathrm{CO}, n) \cong \delta(\mathrm{HC}, n) \cong \delta\left(\mathrm{NO}_{\mathrm{x}}, n\right) \cong 0 .
$$

2) $0.11<v<1 / 5:$ In this interval, while the numbers of particles at rest and with the lower velocities in the system start to be non-negligible in the NS model, all the particles persist in the free flow regime, with the highest velocities in the FI model; however, the number of particles con velocity $v=5$ decreases to zero at $n=1 / 5$. Because of the velocity distribution (Figure 6 , left column) and of the dependence of emission rate on velocity (Figure 8, right column), the emission rates, in this interval, also reach their highest values for each pollutant 


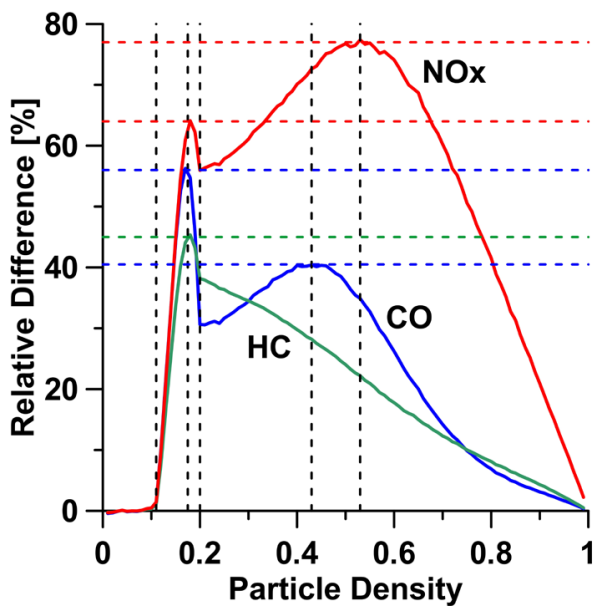

Figure 14. Relative difference (\%) between the FI and NS estimations of the emission rates.

(Figure 11), with larger values for the FI model than for the NS model. Here, all the relative differences $\delta(\mathrm{HC}, n), \delta(\mathrm{CO}, n)$ and $\delta\left(\mathrm{NO}_{\mathrm{x}}, n\right)$ show sharp peaks $(45.36 \%, 56.27 \%$ and $64.10 \%$, respectively) around $n=0.175$, just where the highest values of the FI emission rates occur.

3) $1 / 5<n<1$ : In this interval, both models exhibit a congested flow regime. As the particle density increases, the numbers $n_{4}$ and $n_{5}$ of the particles that move with the highest velocities, decrease monotonically; the number of particles at rest $\left(n_{0}\right)$ increases monotonically; and the numbers of particles with velocities $v_{1}, v_{2}$ and $v_{3}$ grow up to a maximum and then drop to zero. Because of this, the total emission rates diminish monotonically up to their idle condition values, when all the particles become at rest. On the other hand, the relative difference between the emission rates of the FI and NS models (Figure 14) seems to decrease monotonically for hydrocarbons, but for carbon monoxide and nitrogen oxides grows up and then drops to zero, reaching their maximum values, $40.41 \%$ and $76.87 \%$, at $n=0.43$ and $n=0.55$, respectively.

\section{Concluding Remarks}

There exists a growing interest in using cellular automata to model traffic flow phenomena from a microscopic standpoint. The possibility of using these models to simulate traffic in the cities brings out the attention to the problem of assessing the contributions of this phenomenon to the urban air pollution. To do it, the velocity distribution of the traffic network has to be known, spatially and temporally disaggregated. It is also required the engine's emission factors or emission rates as functions of the vehicle velocity. In this work, we used computer simulations and a maximum entropy approach for obtaining the velocity distributions of the traffic cellular automata of Nagel-Schreckenberg and FukuiIshibashi under steady state conditions. The engine emissions were obtained from data available in [18] [33] [34] [35], which allowed us to estimate and compare the emission rates of $\mathrm{CO}, \mathrm{HC}$, and $\mathrm{NO}_{\mathrm{x}}$ produced by the NS and FI 
traffic models.

Although the dynamical rules of the NS and FI models are not microscopically reversible and, therefore, these systems are always far from equilibrium, our estimations of the total traffic emission rates with the maximum entropy velocity distributions resulted very similar to those we obtained using the velocity distributions from computer simulations with these traffic cellular automata.

In general, the emission rates in the FI traffic flow resulted larger than in the NS model. The relative differences $\delta(\alpha, n)$ reached values of up to $45 \%$ in $\mathrm{HC}$, $56 \%$ in $\mathrm{CO}$, and $77 \%$ in $\mathrm{NO}_{\mathrm{x}}$. These results are consequences of the differences between the FI and NS dynamic rules: In the NS model, the acceleration of the particles is gradual, while in the FI model, a particle can accelerate from rest up to the maximum velocity in a single time step. Moreover, the stochastic delay is applied only to the particles with the highest velocities in the FI model.

The ideas of this study can be extended easily to other 1D or 2D traffic cellular automata for estimating the traffic flow contributions to air pollution.

\section{Acknowledgements}

We thank Ana Teresa Celada Murillo (Instituto Nacional de Electricidad y Energías Limpias) for beneficial comments and for her help to make the paper more comprehensible.

\section{Fund}

This research did not receive any specific grant from funding agencies in the public, commercial, or not-for-profit sectors.

\section{Competing Interest Statement}

The authors declare no conflict of interest.

\section{References}

[1] INEGI (2015) Instituto Nacional de Estadística y Geografía (INEGI) [Motor Vehicles Registered in Circulation. Administrative Records of the National Institute of Statistics and Geography.] Data available at the website:

http://www.inegi.org.mx/est/contenidos/proyectos/registros/economicas/vehiculos (in Spanish). Last Access: November 3, 2017.

[2] IE-CDMX (2016) Inventario de Emisiones de la CDMX 2014. Contaminantes Criterio, Tóxicos y de Efecto Invernadero. Secretaría del Medio Ambiente del Gobierno de la Ciudad de México. [Emission Inventory of Mexico City 2014. Criteria, Toxic, and Greenhouse Effect Pollutants.] (in Spanish)

[3] Prigogine, I. and Herman, R.C. (1971) Kinetic Theory of Vehicular Traffic. 1st Edition (July 23, 1971), Elsevier, New York, London, Amsterdam.

[4] Cremer, M. and Ludwig, J. (1986) A Fast Simulation Model for Traffic Flow on the Basis of Boolean Operations. Mathematics and Computers in Simulation, 28, 297-303. https://doi.org/10.1016/0378-4754(86)90051-0

[5] Nagel, K. and Schreckenberg, M. (1992) A Cellular Automaton Model for Freeway 
Traffic. Journal de Physique Archives, 2, 2221-2229.

https://doi.org/10.1051/jp1:1992277

[6] Fukui, M. and Ishibashi, Y. (1996) Traffic Flow in 1D Cellular Automaton Model Including Cars Moving with High Speed. Journal of the Physical Society of Japan, 65, 1868-1870. https://doi.org/10.1143/JPSJ.65.1868

[7] Wagner, P., Nagel, K. and Wolf, D.E. (1997) Realistic Multi-Lane Traffic Rules for Cellular Automata. Physica A, 234, 687-698.

[8] Nagel, K., Wolf, D.E., Wagner, P. and Simon, P. (1998) Two-Lane Traffic Rules for Cellular Automata: A Systematic Approach. Physical Review E, 58, 1425-1437. https://doi.org/10.1103/PhysRevE.58.1425

[9] Chowdhury, D., Santen, L. and Schadschneider, A. (2000) Statistical Physics of Vehicular Traffic and Some Related Systems. Physics Reports, 329, 199-329.

[10] Rickert, M. and Nagel, K. (1997) Experiences with a Simplified Microsimulation for the Dallas/Fort Worth Area. International Journal of Modern Physics C, 8, 483-503.

[11] Nagel, K. and Barrett, C.L. (1997) Using Microsimulation Feedback for Trip Adaptation for Realistic Traffic in Dallas. International Journal of Modern Physics C, 8 , 505-525.

[12] Tonguz, O.K. and Viriyasitavat, W. (2009) Modeling Urban Traffic: A Cellular Automata Approach. IEEE Communications Magazine, May 2009, 142-150. https://doi.org/10.1109/MCOM.2009.4939290

[13] Zhou, T. and Lijuan, P. (2013) Cellular Automata Simulation of Urban Traffic Flow Considering Bus Lane. International Journal of Online Engineering, 9, 65-70. https://doi.org/10.3991/ijoe.v9iS7.3193

[14] Kurnaz, İ. (2016) Urban Traffic Modeling with Microscopic Approach using Cellular Automata. Technical Gazette, 23, 1565-1570.

[15] Salcido, A. (2007) The Maximum Entropy States of 1D Cellular Automata Traffic Models. Proceedings of the 18 th IASTED International Conference on Modelling and Simulation, Montreal, 30 May-1 June 2007, 160-165.

[16] Salcido, A. (2011) Equilibrium Properties of the Cellular Automata Models for Traffic Flow in a Single Lane. In: Salcido, A., Ed., Cellular Automata, Simplicity behind Complexity, InTech, 159-192. https://doi.org/10.5772/15371

[17] Salcido, A., Hernández-Zapata, E. and Carreón-Sierra, S. (2017) Exact Results of 1D Traffic Cellular Automata: The Low-Density Behavior of the Fukui-Ishibashi Model. (under Review in Physica A)

[18] HBEFA (1999) Handbuch Emissionsfaktoren des Straßenverkehrs. [Handbook of Emission Factors of Road Traffic.] Version 1.2, on Behalf of the Swiss Ministry of Environment, Forestry and Agriculture, Bern and the German Environmental Agency Berlin. (in German)

[19] Von Neumann, J. (1966) The Theory of Self-Reproducing Automata. University of Illinois Press, Urbana.

[20] Ilachinski, A. (2001) Cellular Automata. A Discrete Universe. World Scientific, Singapore.

[21] Salcido, A. (2011) Cellular Automata. Simplicity behind Complexity. InTech.

[22] Salcido, A. (2011) Cellular Automata. Innovative Modelling for Science and Engineering. InTech.

[23] Salcido, A. (2013) Emerging Applications of Cellular Automata. InTech.

[24] Wolfram, S. (1984) Computation Theory of Cellular Automata. Communications in 
Mathematical Physics, 96, 15-57.

[25] Gardner, M. (1970) The Fantastic Combinations of John Conway's New Solitaire Game "Life". Scientific American, 223, 120.

[26] Fukui, M. and Ishibashi, Y. (1993) Evolution of Traffic Jam in Traffic Flow Model. Journal of the Physical Society of Japan, 62, 3841-3844. https://doi.org/10.1143/JPSJ.62.3841

[27] Schadschneider, A. (1999) The Nagel-Schreckenberg Model Revisited. The European Physical Journal B, 10, 573-582. https://doi.org/10.1007/s100510050888

[28] Brilon, W. and Wu, N. (1999) Evaluation of Cellular Automata for Traffic Flow Simulation on Freeway and Urban Streets. In: Brilon, W., Huber, F., Schreckenberg, M. and Wallentowitz, H., Eds., Traffic and Mobility, Springer, Berlin, Heidelberg.

[29] Wagner, P., Nagel, K. and Wolf, D.E. (1997) Realistic Multi-Lane Traffic Rules for Cellular Automata. Physica A, 234, 687-698.

[30] Klein, M.J. (1955) Principle of Detailed Balance. Physical Review, 97, 1446-1447. https://doi.org/10.1103/PhysRev.97.1446

[31] Gorban, A.N. (2014) Detailed Balance in Micro- and Macrokinetics and Micro-Distinguish Ability of Macro-Processes. Results in Physics, 4, 142-147.

[32] Schütz, G.M. (2001) Exactly Solvable Models for Many-Body Systems Far from Equilibrium. In: Domb, C. and Lebowitz, J.L., Eds., Phase Transitions and Critical Phenomena, Vol. 19, Academic Press, San Diego, 1-251.

[33] Walther, C. (2000) Geschwindigkeiten und Schadstoffemissionen des motorisierten Straßenverkehrs in innerörtlichen Netzen auf der Grundlage der EWS-Berechnungsvorschriften. [Speeds and Pollutant Emissions of Motorized Road Transport in Intra-Urban Networks Based On EWS (Environmental Protection) Calculation Rules.] Straßenverkehrstechnik, 1, 19-25. (in German)

[34] Eissfeldt, N.G. (2004) Vehicle-Based Modelling of Traffic. Theory and Application to Environmental Impact Modelling. Inaugural-Dissertation zur Erlangung des Doktorgrades der Mathematisch-Naturwissenschaftlichen Fakultät der Universität zu Köln. [Vehicle-Based Modelling of Traffic. Theory and Application to Environmental Impact Modelling. Inaugural-Dissertation to Obtain the Doctor Degree of the Faculty of Mathematics and Natural Sciences of the University of Cologne.]

[35] Eissfeldt, N. and Schrader, R. (2002) Calculation of Street Traffic Emissions with a Queuing Model. Journal of Computational Technologies, 7, 5-15. http://e-archive.informatik.uni-koeln.de/id/eprint/412 